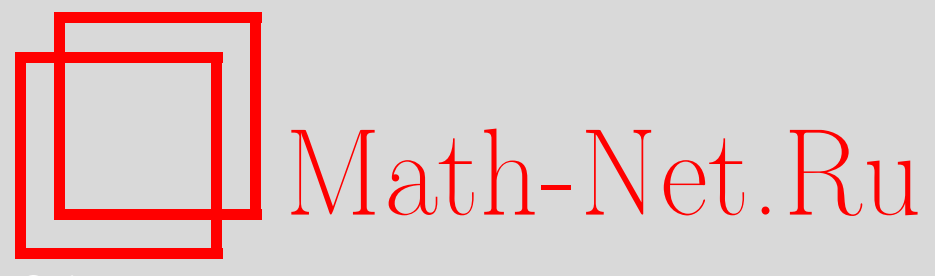

В. П. Радченко, А. Ю. Куров, Влияние анизотропии поверхностного пластического упрочнения на формирование остаточных напряжений в цилиндрических деталях с надрезами полукруглого профиля, Вестн. Сам. гос. техн. ун-та. Сер. Физ.мат. науки, 2016, номер 4, 675-690

DOI: https://doi.org/10.14498/vsgtu1513

Использование Общероссийского математического портала MathNet.Ru подразумевает, что вы прочитали и согласны с пользовательским соглашением

http://www.mathnet.ru/rus/agreement

Параметры загрузки:

IP: 18.209 .158 .208

26 апреля 2023 г., 13:48:38

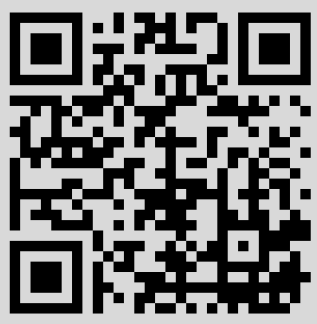


УДК 539.43:621.787

\title{
ВЛИЯНИЕ АНИЗОТРОПИИ ПОВЕРХНОСТНОГО ПЛАСТИЧЕСКОГО УПРОЧНЕНИЯ НА ФОРМИРОВАНИЕ ОСТАТОЧНЫХ НАПРЯЖЕНИЙ В ЦИЛИНДРИЧЕСКИХ ДЕТАЛЯХ С НАДРЕЗАМИ ПОЛУКРУГЛОГО ПРОФИЛЯ
}

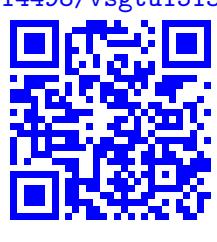

\author{
В. П. Радченко, А. Ю. Куров
}

Самарский государственный технический университет, Россия, 443100, Самара, ул. Молодогвардейская, 244.

\section{Аннотация}

Исследуется влияние анизотропии поверхностного пластического упрочнения на формирование остаточных напряжений в сплошных цилиндрических образцах и образцах с полукруглым надрезом. В качестве исходной информации используется одна и/или две экспериментально определенные компоненты остаточных напряжений в упрочненном слое гладкого образца. Изложена расчетная методика остальных диагональных компонент тензоров остаточных напряжений и пластических деформаций, недиагональные компоненты не рассматриваются. Предложен численный метод расчета остаточных напряжений в концентраторе напряжений цилиндрического образца после опережающего поверхностного пластического деформирования. Задача сведена к краевой задаче фиктивной термоупругости, где начальные (пластические) деформации моделируются температурными деформациями. Решение построено с использованием метода конечных элементов. Детально исследовано влияние радиуса надреза и параметра анизотропии упрочнения на характер и величину распределения остаточных напряжений по глубине слоя в наименьшем сечении цилиндрических образцов из сплава ЭИ961 и стали 45. Установлено, что при малых радиусах надреза, меньших толщины упрочненного слоя, величина осевой компоненты остаточных напряжений (по модулю) в концентраторе больше, чем в гладком образце. Выполнена экспериментальная проверка разработанного метода для гладких образцов и установлено соответствие расчетных и экспериментальных данных о распределении осевых и окружных остаточных напряжений по глубине упрочненного слоя. Для образцов с концентраторами построенные численные решения сравниваются с известными решениями других авторов.

Ключевые слова: поверхностно пластическое упрочнение, анизотропия, цилиндрический образец, остаточные напряжения, полукруглый надрез, краевая задача, метод конечных элементов.

(C) 2016 Самарский государственный технический университет.

Образец для цитирования

Р адченко В. П., Куров А. Ю. Влияние анизотропии поверхностного пластического упрочнения на формирование остаточных напряжений в цилиндрических деталях с надрезами полукруглого профиля // Вестн. Сам. гос. техн. ун-та. Сер. Физ.-мат. науки, 2016. T. 20, № 4. C. 675-690. doi: 10.14498/vsgtu1513.

\section{Сведения об авторах}

Владимир Павлович Радченко (д.ф.-м.н., проф.; radch@samgtu.ru; автор, ведущий переписку), заведующий кафедрой, каф. прикладной математики и информатики.

Алексей Юръевич Куров (alexeykurov@gmail.com), аспирант, каф. прикладной математики и информатики. 
1. Постановка задачи. Особое место в решении проблемы повышения ресурса деталей и элементов конструкций играют технологические методы, связанные с процедурами поверхностного пластического деформирования, к которым относятся пневмодробеструйная обработка поверхности микрошариками, обкатка роликом, алмазное выглаживание, ультразвуковое упрочнение, термопластическое упрочнение, обработка поверхности импульсным лазерным источником и многие другие. Исследования этих методов для повышения сопротивления усталости, износостойкости и микротвердости выполнялись в ряде работ отечественных [1-7] и зарубежных исследователей [8-13]. Положительное влияние упрочняющих технологий связывают с образованием в тонком приповерхностном слое детали области сжимающих остаточных напряжений. В частности, повышение сопротивления усталости напрямую связывается с интегральными характеристиками распределения остаточных напряжений по глубине слоя [2,3]. Поэтому возникает задача моделирования напряженно-деформированного состояния в упрочненной детали после процедур технологического воздействия. Однако подавляющее большинство работ в этом направлении носит, во-первых, сугубо экспериментальный характер, во-вторых, эти методы позволяют определить одну или две компоненты тензора остаточных напряжений. Определить остаточные пластические деформации экспериментально невозможно. А без начального напряженнодеформированного состояния (НДС), образованного после процедуры упрочнения, нельзя решать задачи механики в условиях воздействия на упрочненную деталь внешних воздействий, когда на начальное НДС накладывается НДС от действия «рабочих» нагрузок (задачи многоциклового нагружения, ползучесть и т. д.).

На практике в явном виде первоначальных (пластических) деформаций не имеется, так как процесс разгрузки протекает одновременно с их формированием. Возникает сложная и не решенная до сих пор в полном объеме задача динамики и упругопластичности в поле силовых и температурных нагрузок, структурно-фазовых превращений в металле и других факторов. Первые попытки решения такого рода задач связаны с волевым заданием закона распределения пластической деформации по пространственным координатам в тонком приповерхностном слое (равномерное распределение, линейный закон по глубине слоя и т. д.). Для некоторых простейших законов распределения пластической деформации в гладких цилиндрических изделиях и плоских образцах в [1] получены аналитические решения. Этот метод расчета остаточных напряжений по заданным первоначальным деформациям получил свое развитие и для цилиндрических и плоских деталей с концентраторами напряжений в виде надрезов различной конфигурации в работах [14-18], где методом конечных элементов исследовалось влияние зоны задания пластических деформаций, их протяженности, закона распределения на формирование поля остаточных напряжений в области концентратора напряжений. Модификация этого метода выполнена в работах $[17,18]$, где проведена аналогия между остаточными пластическими деформациями и температурными деформациями в неоднородном температурном поле. Использование этой связи первоначальных деформаций с термоупругостью материала позволяет применять современные расчетные комплексы, реализующие метод конечных элементов в форме перемещений, при решении задач о распределении оста- 
точных напряжений в поверхностно упрочненных деталях. При этом в $[17,18]$ сделана попытка использовать информацию о распределении одной или двух компонент тензора остаточных напряжений, определенных экспериментально. Для этой цели «подбирается» (численно, итерационным методом) такое температурное поле, что температурные деформации, вызванные неоднородным температурным полем, дают наиболее близкое распределение компонент остаточных напряжений к экспериментально определенным. Однако при таком подходе теряется часть информации о компонентах тензора остаточных напряжений и искажается тензор остаточных пластических деформаций.

В работах [19-21] предложен феноменологический метод расчета распределения компонент тензора остаточных напряжений и пластических деформаций по глубине упрочненного слоя после процедуры поверхностного пластического упрочнения гладких цилиндрических изделий. Поскольку методика, изложенная в [19-21], является основной для решения последующих задач, приведем ее ключевые моменты. Необходимой информацией для реализации этой методики является наличие одной или двух экспериментально определенных компонент тензора остаточных напряжений в упрочненном слое. В цилиндрической системе координат $r, \theta, z$ это окружная и/или осевая компоненты. Недиагональными компонентами тензоров остаточных напряжений и пластических деформаций авторы [19,20] пренебрегали. Для реализации методики используется ряд гипотез, одна из которых следующая: характер распределения пластических деформаций по глубине слоя после процедуры упрочнения такой, что компоненты тензора остаточных пластических деформаций связаны соотношением $q_{z}(r)=\alpha q_{\theta}(r)$, где $q_{z}$ и $q_{\theta}$ - осевая и окружная компоненты тензора остаточных пластических деформаций, $\alpha-$ феноменологический параметр, методика определения которого приведена в $[20,21]$. Значение $\alpha=1$ соответствует изотропному упрочнению поверхности в направлениях осей $z$ и $\theta$ (пневмо- и гидродробеструйная обработка детали, термопластическое и ультразвуковое упрочнение), при $\alpha \neq 1$ имеет место так называемое неизотропное упрочнение (обкатка роликом, алмазное выглаживание, дорнование). В предположении, что в сплошном упрочненном цилиндрическом образце после упрочнения не возникают вторичные пластические деформации в области сжатия, в [20,21] получены следующие соотношения для полей остаточных напряжений и пластических деформаций:

$$
\begin{gathered}
\sigma_{r}(r)=\frac{1}{r} \int_{0}^{r} \sigma_{\theta}(\xi) d \xi, \quad 0 \leqslant r \leqslant R \\
q_{\theta}(r) \frac{(1+\mu)(1-2 \mu)}{E(1+\alpha \mu)^{2}} r^{-\frac{2+\alpha}{1+\alpha \mu}} \int_{0}^{r} \xi^{\frac{1+\alpha-\alpha \mu}{1+\alpha \mu}}\left[\sigma_{r}(\xi)+(1+\alpha) \sigma_{\theta}(\xi)\right] d \xi- \\
-\frac{1+\mu}{E(1+\alpha \mu)}\left[(1-\mu) \sigma_{\theta}(r)-\mu \sigma_{r}(r)\right], \\
q_{z}(r)=\alpha q_{\theta}(r), \quad q_{r}(r)=-(1+\alpha) q_{\theta}(r), \\
\varepsilon_{z}^{0}=\frac{2}{R^{2}} \int_{0}^{R} \xi\left\{q_{r}(\xi)-\frac{\mu}{E}\left[\sigma_{r}(\xi)+\sigma_{\theta}(\xi)\right]\right\} d \xi, \\
\sigma_{z}(r)=E\left(\varepsilon_{z}^{0}-q(r)\right)+\mu\left(\sigma_{r}(r)+\sigma_{\theta}(r)\right),
\end{gathered}
$$

где $\mu, E$ - упругие константы материала; $R$ - радиус цилиндра; $\sigma_{r}(r), \sigma_{\theta}(r)$, $\sigma_{z}(r)$ - радиальное, окружное и осевое остаточные напряжения; $q_{r}(r), q_{\theta}(r)$, 
$q_{z}(r)$ - соответствующие остаточные пластические деформации; $\alpha$ - параметр анизотропии упрочнения $(0<\alpha<\infty)$. Из (1)-(5) следует, что все компоненты напряжений и деформаций выражаются через $\sigma_{\theta}(r)$ и параметр $\alpha$. Поэтому, если процедура упрочнения изотропная $(\alpha=1)$, то из экспериментов достаточно знать лишь распределение $\sigma_{\theta}=\sigma_{\theta}(r)$, если же процедура упрочнения анизотропная, то $\alpha \neq 1$, но этот параметр à priori неизвестен. Поэтому необходимо иметь две экспериментально определенные компоненты: $\sigma_{\theta}=\sigma_{\theta}(r)$ и $\sigma_{z}=\sigma_{z}(r)$, а величина $\alpha$ определяется расчетным путем (способ идентификации $\alpha$ приведем ниже).

Для реализации расчетной схемы (1)-(5) необходимо, чтобы величина $\sigma_{\theta}(r)$ была известна по всему радиусу образца, но экспериментально ее можно определить только в тонком упрочненном слое (области сжатия), поэтому данные для $\sigma_{\theta}(r)$ необходимо экстраполировать в область растяжения, но так, чтобы не нарушалось условие самоуравновешенности для компоненты $\sigma_{\theta}=\sigma_{\theta}(r)$ :

$$
\int_{0}^{R} \sigma_{\theta}(r) d r=0,
$$

которое следует из условия равновесия

$$
r \frac{d \sigma_{r}}{d r}+\sigma_{r}=\sigma_{\theta}
$$

и граничного условия $\sigma_{r}(R)=0$. Для этой цели используется следующая аналитическая аппроксимация компоненты $\sigma_{\theta}(r)$ :

$$
\sigma_{\theta}(r)=\sigma_{0}-\sigma_{1} \exp \left(-\left(R-h^{*}-r\right)^{2} / b^{2}\right), \quad 0 \leqslant r \leqslant R
$$

где $h^{*}=R-r^{*}$ - глубина слоя, при которой компонента $\sigma_{\theta}(r)$ принимает свой минимум; $\sigma_{0}, \sigma_{1}$ и $b$ - параметры, подлежащие определению.

Рассмотрим алгоритм определения параметров $\sigma_{0}, \sigma_{1}$ и $b$ в $(6)$ в предположении, что известна экспериментальная информация для компоненты $\sigma_{\theta}(r)$ в области сжатия и задан параметр анизотропии $\alpha$ в (1). Используя условие самоуравновешенности для окружной компоненты $\sigma_{\theta}(r)$ и условия

$$
\sigma_{\theta}\left(r^{*}\right)=\sigma^{*}, \quad \sigma_{\theta}\left(r_{0}\right)=0
$$

где $\sigma^{*}$ - экспериментальное значение минимума величины $\sigma_{\theta}(r)$, которое достигается в точке $r=r^{*}$, а $r_{0}$ - значение радиуса, при котором значение окружной компоненты обращается в нуль, с учетом (6) получаем систему трех нелинейных уравнений относительно $\sigma_{0}, \sigma_{1}$ и $b$, численное решение которой не вызывает принципиальных трудностей.

Таким образом, методику определения полей остаточных напряжений и пластических деформаций при известных $\alpha$ и экспериментальной зависимости $\sigma_{\theta}=\sigma_{\theta}(r)$ можно представить следующей схемой (над стрелками указаны формулы, по которым определяются соответствующие величины):

$$
R, r_{0}, r^{*}, \sigma^{*} \rightarrow \sigma_{0}, \sigma_{1}, b \stackrel{(6)}{\longrightarrow} \sigma_{\theta}(r) \stackrel{(1)}{\longrightarrow} \sigma_{r}(r) \stackrel{(2)}{\longrightarrow} q_{\theta}(r) \stackrel{(3)}{\longrightarrow} q_{r}, q_{z} \stackrel{(4),(5)}{\longrightarrow} \sigma_{z}(r),
$$


при этом в схеме $(7)$ под функцией $\sigma_{\theta}=\sigma_{\theta}(r)$ подразумевается уже аналитическая аппроксимация этой зависимости по формуле (6).

Однако на практике величина $\alpha$ в соотношении (1) неизвестна, и ее можно определить лишь после проведения экспериментальных исследований. В этом случае схема (7) существенно изменяется, и в качестве исходной экспериментальной информации необходимо иметь экспериментальные зависимости $\sigma_{\theta}(r)$ и $\sigma_{z}(r)$ по толщине упрочненного слоя, а параметр $\alpha$ подлежит процедуре идентификации. Здесь задача сводится к поисковому методу оптимизации: целенаправленно варьируются параметры $r_{0}, r^{*}, \sigma^{*}$, которые, в свою очередь, варьируют параметры аппроксимации (6), и $\alpha$, и для каждой такой совокупности значений этих параметров осуществляется численный расчет по схеме (7) до достижения минимума функционала:

$$
\Delta_{\theta}+\Delta_{z} \rightarrow \min ; \quad \Delta_{i}=\left[\frac{\sum_{k=1}^{N}\left(\sigma_{i}\left(r_{k}\right)-\tilde{\sigma}_{i}\left(r_{k}\right)\right)^{2}}{\sum_{k=1}^{N}\left(\tilde{\sigma}_{i}\left(r_{k}\right)\right)^{2}}\right]^{1 / 2}, \quad i=\theta, z,
$$

где $\Delta_{i}$ - функционал нормированного среднеквадратического отклонения расчетных значений $\sigma_{i}\left(r_{k}\right)$ от соответствующих экспериментальных значений $\tilde{\sigma}_{i}\left(r_{k}\right) ; N$ - число точек дискретизации радиуса $r_{k}$ в упрочненном слое, в которых известны экспериментальные и расчетные значения окружной и осевой компонент остаточных напряжений.

Отметим, что традиционные технологии упрочнения, так же как и изложенный метод моделирования напряженно-деформированного состояния в упрочненном слое, применимы для гладких деталей. Однако многие цилиндрические изделия имеют концентраторы напряжений в виде надрезов различной геометрической конфигурации, наличие которых обусловлено функциональными потребностями тех или иных конструкторско-технологических решений, и если размеры концентратора малы по сравнению с геометрическими размерами деформирующего элемента или доступ к концентратору ограничен, то стандартные технологии упрочнения не применимы. Поэтому на практике изготовлению мелких концентраторов напряжений предшествует упрочнение гладкой детали (опережающее упрочнение поверхности детали). При таком подходе после упрочнения в детали возникают неоднородное поле остаточных пластических деформаций и неоднородное по глубине залегания поле остаточных напряжений, а после изготовления концентратора напряжений (фактически после удаления части объема) под действием остаточных пластических деформаций, играющих роль начальных деформаций, в упрочненном поверхностном слое происходит перераспределение остаточных напряжений, при этом наиболее интенсивно - в окрестности концентратора. В работах [22,23] предложен метод решения этой задачи на основе конечноэлементного моделирования с использованием распределения полей остаточных напряжений и пластических деформаций для сплошного цилиндра с учетом схемы (7). С использованием этой информации в [22, 23] расчетным путем построены зависимости для остаточных напряжений в концентраторе в наименьшем сечении детали. Целью данной работы является развитие идей этого метода в направлении исследования влияния параметра анизотропии упрочнения $\alpha$ (соотношение (3)) на характер распределения остаточных напряжений в концентраторе напряжения (полукруглого надреза разных размеров). 
2. Численное решение задачи о перераспределении напряжений в концентраторе напряжений сплошного цилиндра после опережающего поверхностного пластического деформирования. Как отмечалось выше, образование остаточных напряжений в упрочненных деталях происходит за счет наведенных неупругих первоначальных деформаций (остаточных пластических деформаций). Очевидно, что компоненты тензора первоначальных деформаций не удовлетворяют условиям совместности. Следовательно, в сплошной среде эти деформации не могут быть реализованы, так как в ней могут существовать только совместные деформации. Поэтому после появления первоначальных (пластических) деформаций возникают препятствующие нарушению условий сплошности внутренние силы, вызывающие самоуравновешенное поле собственных (остаточных) напряжений. Эти напряжения, точнее отдельные компоненты тензора напряжений, на практике «измеряются» различными методами и способами, в основе которых лежит предположение, что остаточные напряжения не превышают предела текучести материала и вызванная ими деформация является упругой.

Таким образом, для решения задачи расчета остаточных напряжений необходимо иметь поле остаточных пластических деформаций такое, чтобы тензор полных деформаций, соответствующий наведенному полю остаточных напряжений, удовлетворял условию совместности деформаций. Ни один из известных экспериментальных методов и расчетных методов на основе информации об остаточных первоначальных деформациях (например, [14-18]) в полной мере не позволяет это сделать. Но это позволяет сделать схема (1)-(5), (6). Для задания поля наведенных остаточных пластических деформаций используется аналогия с объемными температурными деформациями при ортотропном законе распределения температурного поля. Использование этой связи первоначальных (остаточных пластических) деформаций с термоупругостью материала и позволяет использовать современные расчетные комплексы, реализующие метод конечных элементов в форме перемещений, при решении задач о распределении остаточных напряжений в поверхностно упрочненных деталях сложной формы, в том числе-и с концентраторами напряжений. Сведение исходной задачи к задаче термоупругости решает проблему корректности решения соответствующей краевой задачи.

Решение задачи о перераспределении остаточных напряжений в образце с полукруглым надрезом радиуса $\rho$ (см. рис. 1), нанесенным после процедуры опережающего поверхностного пластического деформирования, состоит из следующих этапов. На первом этапе определяются поля остаточных напряжений и пластических деформций после процедуры упрочнения для гладкого цилиндрического образца согласно схеме (1)-(5), (6), (7).

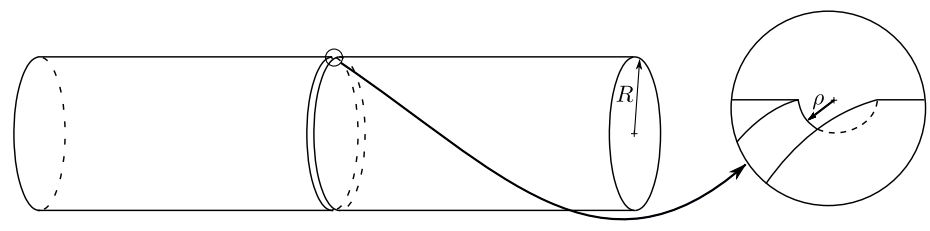

Рис. 1. Схема образца с круговым надрезом [Figure 1. Scheme of sample with semicircular notch] 
На втором этапе аналитически определенные зависимости для компонент тензора остаточных пластических деформаций $q_{i}=q_{i}(r)(i=r, \theta, z)$ моделируются температурными деформациями с использованием соотношений

$$
q_{i}(r)=\beta_{i}(T(r))\left[T(r)-T_{0}\right], \quad i=r, \theta, z, \quad 0 \leqslant r \leqslant R
$$

где $T_{0}=$ const - начальная температура, а закон изменения $T=T(r)$ задается, вообще говоря, любой функцией (в настоящей работе использовалась линейная функция). Тогда, зная $q_{i}(r)$, из $(9)$ определяется функция $\beta_{i}=\beta_{i}(T(r))$ как функция радиуса. В дальнейшем под первоначальными температурными деформациями будем понимать величины $q_{i}=q_{i}(r)$.

На третьем этапе на гладкий образец наносится полукруговой надрез (см. рис. 1), т. е. удаляется часть материала с наведенными остаточными деформациями. Далее строится геометрическая конечно-элементная модель цилиндрического образца с концентратором напряжений с заданными по формуле (9) псевдотемпературными начальными деформациями; точнее, в качестве исходных данных задаются $T=T(r)$ и $\beta_{i}=\beta_{i}(T(r))(i=r, \theta, z)$, которые и задают начальные деформации в соответствии с (9). При построении геометрической модели необходимо учитывать, что остаточные напряжения обладают существенным градиентом в поверхностном слое. Так, на линейном размере в 200-400 мкм вдоль радиуса величины напряжений $\sigma_{z}$ и $\sigma_{\theta}$ изменяются в пределах трех порядков. Это приводит к необходимости создания достаточно мелкой конечно-элементной сетки в области сжатия материала (в приповерхностном слое) с линейным размером конечного элемента 2-3 мкм.

На четвертом этапе стандартными методами на основе метода конечных элементов решается фиктивная задача термоупругости относительно остаточных напряжений (начальные (термоупругие) деформации фактически задаются (9)). При этом, если в гладком образце имелись лишь компоненты $\sigma_{r}$, $\sigma_{\theta}$ и $\sigma_{z}$, то в образце с концентратором (особенно в области, примыкающей к нему) возникнут и недиагональные компоненты тензора напряжений.

3. Результаты расчетов и их анализ. На первом этапе выполнена проверка адекватности модели (1)-(5), (6), (7) экспериментальным данным в условиях изотропного (в соотношении (3) величина $\alpha=1)$ и анизотропного $(\alpha \neq 1)$ упрочнения гладких образцов. На рис. 2, а приведены экспериментальные данные для компоненты $\sigma_{z}=\sigma_{z}(h)(h=R-r)$ [2] при гидродробеструйной обработке (ГДО) цилиндрического образца из стали 45 радиуса $R=5$ мм. В этом случае величина $\alpha=1$. Для вычисления параметров аппроксимации (6) для $\sigma_{\theta}=\sigma_{\theta}(r)$ сначала определялись величины $\sigma_{0}, \sigma_{1}$ и $b$ по экспериментальной диаграмме для $\sigma_{z}=\sigma_{z}(r)$ (при $\alpha=1$ диаграммы $\sigma_{\theta}=\sigma_{\theta}(r)$ и $\sigma_{z}=\sigma_{z}(r)$ близки). Затем эти параметры варьировались, для каждого набора параметров $\sigma_{0}, \sigma_{1}$ и $b$ реализовывалась схема (7) и расчетным путем определялась величина $\sigma_{z}=\sigma_{z}(r)$. Далее осуществлялась минимизация функционала (8) для $\Delta_{z}$. В итоге получены значения $\sigma_{0}=17.82 \mathrm{MПа;} \sigma_{1}=396.4 \mathrm{MПа;}$ $b=167$ мкм, $h_{0}=378$ мкм; $h^{*}=83$ МПа, при которых $\Delta_{z}=0.043$, т. е. отклонение расчетных значений компоненты $\sigma_{z}=\sigma_{z}(r)$ от экспериментальных в норме (8) составляет 4.3\%. На рис. 2, а приведены сплошными линиями расчетные диаграммы $\sigma_{i}=\sigma_{i}(h)(i=r, \theta, z)$ в зависимости от глубины упрочненного слоя $h$, а на рис. 2 , $б$ представлены зависимости остаточных пластических деформаций $q_{i}=q_{i}(h), i=r, \theta, z$. 

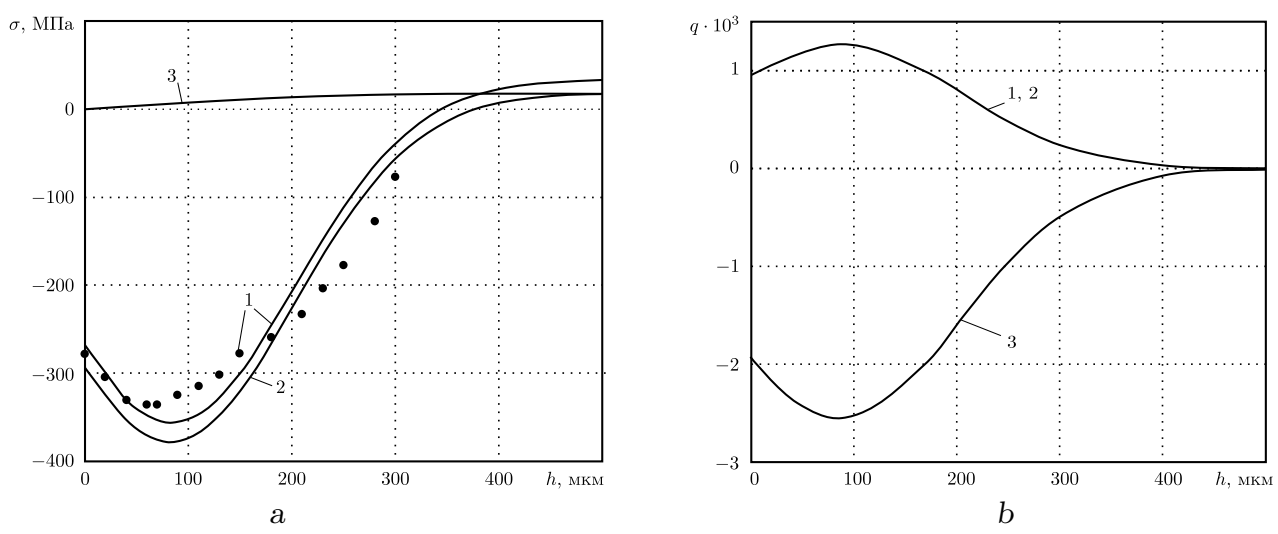

Рис. 2. Сплошной образец из стали $45, R=5$ мм, поля остаточных напряжений (a) и пластических деформаций $(b)$ после ГДО: 1 -осевая компонента $\sigma_{z}, q_{z} ; 2$-окружная компонента $\sigma_{\theta}, q_{\theta} ; 3$ - радиальная компонента $\sigma_{r}, q_{r}$; значки - эксперимент; сплошные линии-расчет; $\alpha=1$ [Figure 2. Solid steel 45 sample, $R=5 \mathrm{~mm}$, residual stresses fields (a) plastic strain $(b)$ after hydro stream hardening: 1 - axial component $\sigma_{z}, q_{z} ; 2$ - circumferential component $\sigma_{\theta}, q_{\theta} ; 3$ - radial component $\sigma_{r}, q_{r}$; points - experimental data; solid lines numerical solution; $\alpha=1]$

На рис. 3 приведены экспериментальные значения для осевой и окружной компонент остаточных напряжений в случае анизотропной процедуры упрочнения цилиндрического образца радиуса $R=5$ мм (алмазное выглаживание). Здесь величина $\alpha$ à priori не известна. Поэтому значения параметров аппроксимации (6) определялись по экспериментальной кривой $\sigma_{\theta}=\sigma_{\theta}(r)$ и в результате получено: $\sigma_{0}=18.88 \mathrm{MПа;} \sigma_{1}=451.9$ МПа; $b=236$ мкм при $h_{0}=420 \mathrm{мкм} ; h^{*}=0$. Для определения величины $\alpha$ минимизировался функционал (8) как функция от аргумента $\alpha$. В результате получено $\alpha=14.6$ при величине $\Delta_{z}+\Delta_{\theta}=0.193$. На рис. 3 сплошными линиями проведены расчетные значения остаточных напряжений $\sigma_{i}=\sigma_{i}(h), i=r, \theta, z$. В целом для гладких образцов наблюдается удовлетворительное соответствие расчетных и экспериментальных данных для остаточных напряжений $\sigma_{\theta}$ и $\sigma_{z}$ в случае как изотропного, так и неизотропного упрочнения поверхности.

На втором этапе исследовались остаточные напряжения, возникающие в образцах с концентратором в виде полукруглого надреза радиуса $\rho$ (см. рис. 1), при этом наибольший интерес представляет распределение напряжений по глубине слоя $h=R-\rho-r$ от «дна» концентратора, т. е. в наименьшем сечении детали. Анализировалось влияние радиуса надреза и параметра анизотропии $\alpha$ на характер и величину остаточных напряжений в концентраторе напряжений. На рис. 4 в качестве примера приведены расчетные значения всех трех компонент $\sigma_{i}=\sigma_{i}(h)(i=r, \theta, z)$ при двух значениях радиуса надреза: $\rho=0.3$ мм и $\rho=0.5$ мм, полученные решением фиктивной задачи термоупругости для образца из сплава ЭИ961 (при $\alpha=14.6$ ), а на рис. 5 для тех же радиусов надреза для образцов из стали 45 (при $\alpha=1$ ). Из сравнения данных следует, что для рассматриваемых образцов в концентраторе на дне надреза при малых значениях $\rho$ происходит существенное увеличение значения компоненты $\sigma_{z}=\sigma_{z}(h)$ по сравнению с аналогичной величиной в гладком образце. Кроме этого, происходит инверсия графика $\sigma_{r}=\sigma_{r}(h)$ относительно оси абсцисс с существенным увеличением значений радиальной 


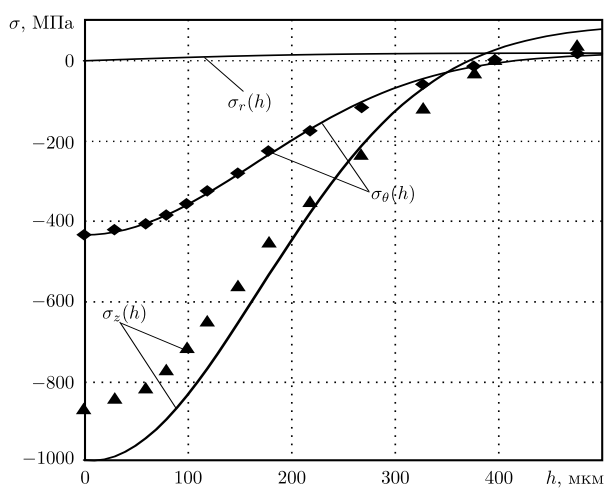

Рис. 3. Расчетные (сплошные линии) и экспериментальные (значки) значения остаточных напряжений по глубине слоя $h$ для гладкого цилиндрического образца из сплава ЭИ961 после алмазного выглаживания [Figure 3. Numerical (solid lines) and experimental (marks) values of residual stresses depending on depth $h$ of cylindrical sample of alloy EI961 without notches after diamond burnishing]

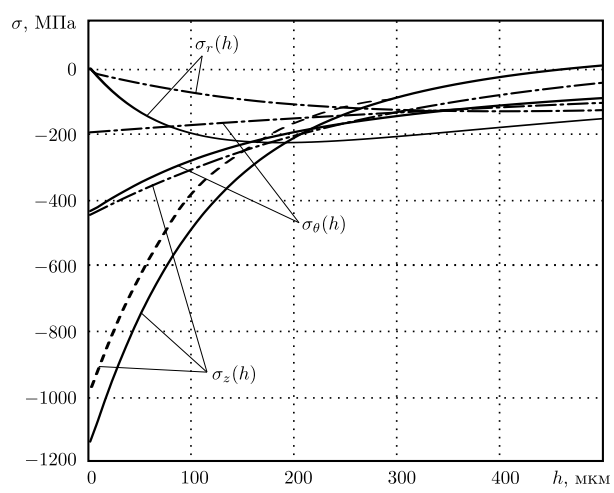

Рис. 4. Распределения остаточных напряжений от дна надреза по толщине слоя $h$ в наименьшем сечении детали из сплава ӘИ961: сплошная линия - надрез $\rho=0.3$ мм; штрихпунктирная линия - надрез $\rho=0.5$ мм; штриховая линия-расчет по методике [4] [Figure 4. Distribution of residual stresses from the bottom of notch in the smallest cross section of detail from alloy EI961 depending on depth $h$ : solid line - notch radius is $0.3 \mathrm{~mm}$, dot-dashed line - notch radius is $0.5 \mathrm{~mm}$, dashed line - method [4] solution]

компоненты напряжений (по модулю) по сравнению с гладким образцом (см. рис. 2). В то же время напряжение $\sigma_{\theta}=\sigma_{\theta}(h)$ становится несколько меньше (по модулю) на дне надреза по отношению к этому напряжению в гладком образце.

Для сопоставимости результатов выполнен сравнительный анализ расчетных значений для напряжения $\sigma_{z}$ по методике настоящей работы с данными расчета этой компоненты по приближенной аналитической зависимости, полученной в [2] методами теории функций комплексного переменного для плоской задачи, когда вместо всего цилиндрического образца рассматривалась тонкая пластина - осевое сечение цилиндра с концентратором напряжений. На рис. 4 данные расчета величины $\sigma_{z}$ по методике [2] приведены штриховой линией, при этом наблюдается соответствие данных расчета по обеим методикам.

Отметим, что остаточное напряжение $\sigma_{z}=\sigma_{z}(h)$ в концентраторе напряжений играет ключевую роль в теории влияния остаточных напряжений на предел сопротивления усталости $[2,3,14]$, поскольку она входит в соответствующие критериальные зависимости.

Второй задачей параметрического анализа являлось исследование величины параметра $\alpha$ на распределение остаточных напряжений в цилиндрических образцах с надрезами. В качестве модельной использовалась цилиндрическая деталь из стали 45 при двух значениях $\rho=\{0.3 ; 0.5\}$ мм. В исследованиях полагалось, что распределение $\sigma_{\theta}=\sigma_{\theta}(h)$ в гладком образце одно и то же и соответствует величине $\alpha=1$ (см. рис. 2). Величина $\alpha$ варьировалась, в расчетах она принимала следующие значения: $\alpha=\{0.25 ; 0.5 ; 0.75 ; 2 ; 4 ; 8\}$. При каждом значении $\alpha$ и одном и том же распределении $\sigma_{\theta}=\sigma_{\theta}(r)$ по схеме 
(7) рассчитывались величины $\sigma_{z}, \sigma_{r}, q_{r}, q_{\theta}$ и $q_{z}$. Затем по изложенной выше методике численно решалась краевая задача фиктивной термоупругости уже для изделия с концентратором. На рис. 6 и 7 приведены распределения $\sigma_{z}=\sigma_{z}(h)$ как в гладком образце, так и в образцах с концентраторами при радиусах надреза $\rho=0.3$ мм и $\rho=0.5$ мм. Как следует из этих рисунков, наблюдается существенное влияние величины $\alpha$ на характер и величину остаточных напряжений $\sigma_{z}=\sigma_{z}(h)$ и для гладкого образца, и в наимень-

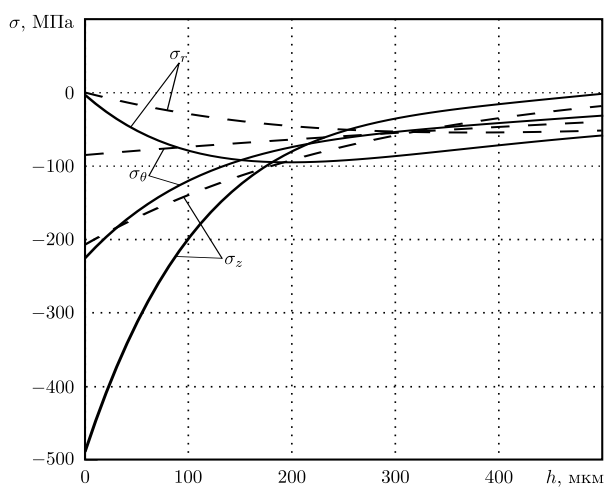

Рис. 5. Распределения остаточных напряжений от дна надреза по толщине слоя $h$ в наименьшем сечении детали из стали 45 при параметре анизотропии $\alpha=1$ : сплошная линия - надрез $\rho=0.3$ мм; штриховая линия - надpe3 $\rho=0.5 \mathrm{~mm}$ [Figure 5. Distribution of residual stresses from the bottom of notch in the smallest cross section of detail from steel 45 depending on depth $h$; solid line notch radius is $0.3 \mathrm{~mm}$, dashed line - notch radius is $0.5 \mathrm{~mm}$ ]

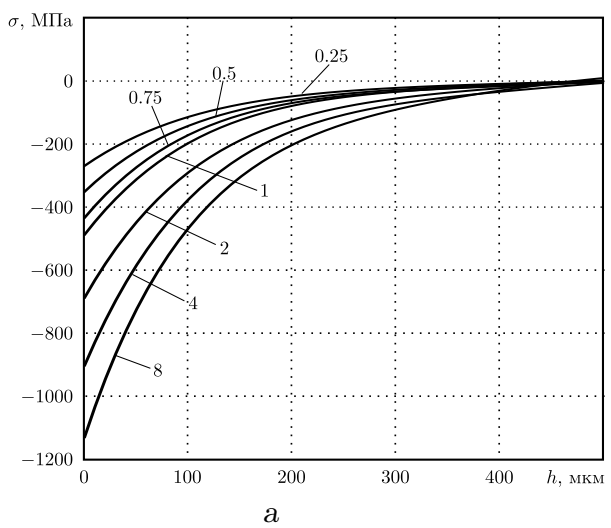

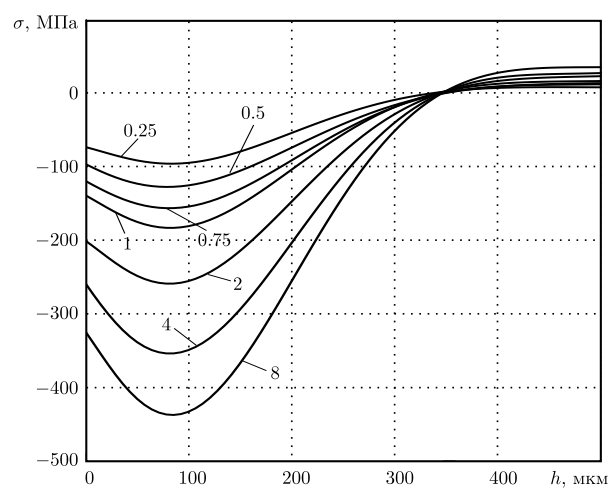

Рис. 6. Эпюры остаточных напряжений $\sigma_{z}=\sigma_{z}(h)$ для гладкого образца из стали 45 при различных значениях параметра анизотропии. Цифры: значения параметра $\alpha$ [Figure 6. Diagrams of residual stresses for sample without notches from steel 45 with different values of anisotropy parameter. Numbers - values of anisotropy parameter $\alpha$ ]

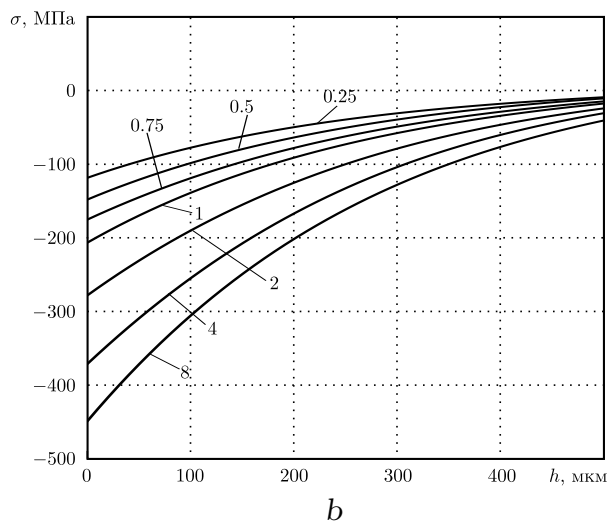

Рис. 7. Эпюры остаточный напряжений $\sigma_{z}=\sigma_{z}(h)$ от дна надреза по толщине слоя $h$ в наименьшем сечении детали из стали 45 при различных значениях параметра анизотропии: $a-\rho=0.3$ мм; б) - $\rho=0.5$ мм. Цифры: значения параметра $\alpha$ [Figure 7. Diagrams of residual stresses from the bottom of notch $\mathrm{n}$ the smallest cross section of detail from steel 45 depending on depth $h$ with different values of anisotropy parameter. Numbers - values of anisotropy parameter $\alpha$ ] 
шем сечении детали с концентратором (при одной и той же эпюре $\sigma_{\theta}=\sigma_{\theta}(h)$ в гладком образце).

Анализ зависимостей на рис. 6 и рис. 7 свидетельствует, во-первых, что для мелкого надреза при $\rho=0.3$ мм, глубина которого меньше упрочненного слоя (области сжимающих напряжений), происходит увеличение (по модулю) величины $\sigma_{z}=\sigma_{z}(h)$ по сравнению с гладким образцом. Если же глубина надреза превосходит толщину упрочненного слоя (при $\rho=0.5 \mathrm{MM}$ ), абсолютное значение $\sigma_{z}=\sigma_{z}(h)$ меньше соответствующей величины в гладком образце. Во-вторых, управление технологией упрочнения (обеспечение заданного значения параметра анизотропии) теоретически позволяет существенно увеличить (по модулю) компоненту остаточных напряжений $\sigma_{z}=\sigma_{z}(h)$, которая играет ключевую роль, например, в критериальных зависимостях при оценке увеличения предела сопротивления усталости $[2,3,14]$ после процедуры упрочнения.

4. Заключение. Результаты выполненных исследований позволяют сформулировать следующие выводы.

1. Разработан численный метод решения краевой задачи о перераспределении остаточных напряжений в концентраторе цилиндрического образца после опережающего поверхностного пластического упрочнения при заданных начальных (псевдотемпературных, соответствующих пластическим) деформациях.

2. Выполнены исследования влияния анизотропии поверхностного пластического упрочнения гладкого образца на последующее распределение остаточных напряжений в концентраторе после нанесения надреза. Показано, что при значениях параметра анизотропии $0<\alpha<1$ происходит уменьшение (по модулю) компонент тензора остаточных напряжений $\sigma_{\theta}$ и $\sigma_{z}$ в детали с концентратором по сравнению со случаем изотропного упрочнения $(\alpha=1)$, а при $\alpha>1-$ происходит увеличение (по модулю) этих компонент.

3. Установлено, что если глубина надреза меньше упрочненного слоя (области сжатия материала), происходит увеличение (по модулю) величины остаточных напряжений $\sigma_{z}=\sigma_{z}(h)$, а если глубина надреза больше толщины слоя, то уменьшение (по модулю) этой компоненты по сравнению с гладким образцом для всех значений параметра анизотропии упрочнения $\alpha$.

Декларация о финансовых и других взаимоотношениях. Работа выполнена при поддержке Минобрнауки России в рамках базовой части государственного задания ФГБОУ ВО «СамГТУ» (код проекта 1151) и Российского фонда фундаментальных исследований (проект № 16-01-00249-а). Все авторы принимали участие в разработке концепции статьи и в написании рукописи. Авторы несут полную ответственность за предоставление окончательной рукописи в печать. Окончательная версия рукописи была одобрена всеми авторами. Авторы не получали гонорар за статью.

\section{ORCIDs}

Владимир Павлович Радченко: http://orcid.org/0000-0003-4168-9660

Алексей Юрьевич Куров: http://orcid.org/0000-0002-3791-3829

\section{БИБЛИОГРАФИЧЕСКИЙ СПИСОК}

1. Биргер И. А. Остаточные напряжения. М.: Машгиз, 1963. 262 с.

2. Павлов В. Ф., Кирпичев В. А., Иванов В. Б. Остаточные напряжения и сопротивле- 
ние усталости упрочненных деталей с концентраторами напряжений. Самара: СНЦ PAH, 2008. $64 \mathrm{c}$.

3. Иванов С. И., Павлов В. Ф., Минин Б. В., Кирпичев В. А., Кочеров Е. П., Головкин В. В. Остаточные напряжения и сопротивление усталости высоконагруженных резъбовых деталей. Самара: СНЦ РАН, 2015. 170 с.

4. Кравченко Б. А., Круцило В. Г., Гутман Г. Н. Термопластическое упрочнение - резерв повышения прочности и надежности деталей машин. Самара: Самар. гос. техн. ун-т, 2000. 216 c.

5. Сулима Г. Н., Шувалов В. А., Ягодкин Ю. Д. Поверхностный слой и эксплуатаиионные свойства деталей машин. М.: Машиностроение, 1988. 240 с.

6. Марков А. И. Ультразвуковая обработка материалов. М.: Машиностроение, 1989. 237 c.

7. Ножницкий Ю. А., Фишгойт А. В., Ткаченко Р. И., Теплова С. В. Разработка и применение новых методов упрочнения деталей ГТД, основанных на пластическом деформировании поверхностных слоев // Вестник двигателестроения, 2006. № 2. С. 8-16.

8. Brockman R. A., Braisted W. R., Olson S. E., Tenaglia R. D., Clauer A. H., Langer K., Shepard M. J. Prediction and characterization of residual stresses from laser shock peening// International Journal of Fatigue, 2012. vol.36, no.1. pp. 96-108. doi:10.1016/j. ijfatigue.2011.08.011.

9. Dai K., Shaw L. Analysis of fatigue resistance improvements via surface severe plastic deformation// International Journal of Fatigue, 2008. vol.30, no. 8. pp. 1398-1408. doi: 10.1016/j.ijfatigue.2007.10.010.

10. James M. N., Hughes D. J., Chen Z., Lombard H., Hattingh D. G., Asquith D., Yates J. R., Webster P. J. Prediction and characterization of residual stresses from laser shock peening// Engineering Failure Analysis, 2007. vol.14, no.2. pp. 384-395. doi:10.1016/j. engfailanal.2006.02.011.

11. Majzoobi G. H., Azadikhah K., Nemati J. The effects of deep rolling and shot peening on fretting fatigue resistance of Aluminum-7075-T6// Materials Science and Engineering A, 2009. vol. 516, no. 1-2. pp. 235-247. doi: 10.1016/j.msea.2009.03.020.

12. Soady K. A. Life assessment methodologies incoroporating shot peening process effects: Mechanistic consideration of residual stresses and strain hardening: Part 1 - Effect of shot peening on fatigue resistance// Materials Science and Technology (United Kingdom), 2013. vol. 29, no. 6. pp. 637-651. doi: 10.1179/1743284713Y.0000000222.

13. Terres M. A., Laalai N., Sidhom H. Effect of nitriding and shot-peening on the fatigue behavior of $42 \mathrm{CrMo} 4$ steel: Experimental analysis and predictive approach // Materials and Design, 2012. vol. 35. pp. 741-748. doi: 10.1016/j.matdes.2011.09.055.

14. Павлов В. Ф., Столяров А. К., Вакулюк В. С., Кирпичев В. А. Расчет остаточных напряжений в деталях с концентраторами напряжений по первоначальным деформаииям. Самара: СНЦ РАН, 2008. 124 с.

15. Вакулюк В. С. Исследование влияния толщины упрочненного слоя на остаточные напряжения во впадине концентратора методом первоначальных деформаций // Becmн. Сам. гос. техн. ун-та. Сер. Физ.-мат. науки, 2010. №1(20). С. 222-225. doi : 10.14498/ vsgtu782.

16. Сазанов В. П., Чирков А. В., Самойлов В. А., Ларионова Ю. С. Моделирование перераспределения остаточных напряжений в упрочненных цилиндрических образцах при опережающем поверхностном пластическом деформировании // Вестник Самарского государственного аэрокосмическогоо университета, 2011. № 3(27). С. 171-174.

17. Сазанов В. П., Афанасьева О. С., Михалькина С. А., Анохин Д. В. Использование конечно-элементного моделирования при определении первоначальных деформаций в упрочненном поверхностно-упрочненном слое детали / Труды десятой Всероссийской научной конферениии с международным участием (24-28 мая 2016 г.). Часть 1: Математические модели механики, прочности и надежности элементов конструкций / Матем. моделирование и краев. задачи. Самара: СамГТУ, 2016. С. 195-200. 
18. Сазанов В. П., Вакулюк В. С., Михалкина С. А., Сургутанов Н. А., Кочерова Е. Е. Исследование влияния первоначальных радиальных деформаций на распределение остаточных напряжений в поверхностно упрочненном цилиндре // Известия Самарского научного центра Российской академии наук, 2014. Т. 16, № 4. С. 163-167.

19. Радченко В. П., Саушкин М. Н. Ползучесть и релаксация остаточных напряжений в упрочненных конструкииях. М.: Машиностроение-1, 2005. 226 с.

20. Радченко В. П., Саушкин М. Н., Павлов В. Ф. Метод расчета остаточных напряжений и пластических деформаций в цилиндрических образцах с учетом анизотропии процесса поверхностного упрочнения // ПМТФ, 2011. Т. 52, № 2. С. 173-182.

21. Радченко В. П., Павлов В. Ф., Саушкин М. Н. Исследование влияния анизотропии поверхностного пластического упрочнения на распределение остаточных напряжений в полых и сплошных цилиндрических образцах // Вестник ПНИПУ. Механика, 2015. № 1. C. 130-147. doi: 10.15593/perm.mech/2015.1.09.

22. Саушкин М. Н., Куров А. Ю. Конечно-элементное моделирование распределения остаточных напряжений в сплошных упроченных цилиндрических образцах и образцах с полукруглым надрезом // Вестн. Сам. гос. техн. ун-та. Сер. Физ.-мат. науки, 2011. № 3(24). C. $72-78$. doi : 10.14498/vsgtu963.

23. Саушкин М. Н., Куров А. Ю. Анализ напряженного состояния в надрезах полукруглого профиля после опережающего поверхностного пластического деформирования сплошных цилиндрических образцов // Вестн. Сам. гос. техн. ун-та. Сер. Физ.-мат. науки, 2012. №1(26). C. 133-140. doi : 10.14498/vsgtu1039.

Поступила в редакцию 19/X/2016;

в окончательном варианте - 28/XI/2016; принята в печать - 09/XII/2016. 
Vestn. Samar. Gos. Techn. Un-ta. Ser. Fiz.-mat. nauki

[J. Samara State Tech. Univ., Ser. Phys. \& Math. Sci.], 2016, vol. 20, no. 4, pp.675-690

ISSN: 2310-7081 (online), 1991-8615 (print)

doi: http://dx.doi.org/10.14498/vsgtu1513

MSC: 74A10, 74G70, 74D10, 74E10

\title{
EFFECT OF ANISOTROPY OF SURFACE PLASTIC HARDENING ON FORMATION OF RESIDUAL STRESSES IN CYLINDRICAL SAMPLES WITH SEMICIRCULAR NOTCH
}

\author{
V. P. Radchenko, A. Yu. Kurov \\ Samara State Technical University, \\ 244, Molodogvardeyskaya st., Samara, 443100, Russian Federation.
}

\begin{abstract}
We study effect of anisotropy of surface plastic hardening on formation of residual stresses in solid cylindrical samples and samples with semicircular notch. Experimentally determined one and/or two components of residual stresses in a hardened layer are used as an initial information. We describe calculation method for the rest of diagonal components of residual stresses and plastic strains tensors, off-diagonal components are not considered. We propose numerical method for calculation residual stresses in semicircular notch of surface hardened cylindrical sample. This task was reduced to boundary value problem of fictitious thermoelasticity where initial (plastic) strains are modeled with temperature strains. Solution was build with the use of finite element method. We studied in detail the effect of radius of notch and anisotropy parameters of hardening on the nature and magnitude of distribution of residual stresses depending on the depth of layer in the smallest cross section of cylindrical samples of EI961 alloy steel and 45 steel. It was determined that with small radii of notch lower then thickness of hardening layer the value of axial component of residual stresses (absolute value) is higher then in the sample without a notch. Developed method was experimentally verified for samples without notches and the correspondence between calculated and experimental data was determined on distribution of axial and circumferential residual stresses depending on depth of hardening layer. For samples with notches we compare numerical solutions from this work with known solutions of other authors.
\end{abstract}

Keywords: surface plastic hardening, anisotropy, cylindrical sample, residual stresses, semicircular notch, boundary value problem, finite element method.

(C) 2016 Samara State Technical University.

Please cite this article in press as:

Radchenko V. P., Kurov A. Yu. Effect of anisotropy of surface plastic hardening on formation of residual stresses in cylindrical samples with semicircular notch, Vestn. Samar. Gos. Tekhn. Univ., Ser. Fiz.-Mat. Nauki [J. Samara State Tech. Univ., Ser. Phys. \& Math. Sci.], 2016, vol. 20, no. 4, pp. 675-690. doi: 10.14498/vsgtu1513. (In Russian)

\section{Authors Details:}

Vladimir P. Radchenko (Dr. Phys. \& Math. Sci.; radch@samgtu.ru; Corresponding Author), Head of Dept., Dept. of Applied Mathematics \& Computer Science.

Aleksey Yu. Kurov (alexeykurov@gmail.com), Postgraduate Student, Dept. of Applied Mathematics \& Computer Science. 
Declaration of Financial and Other Relationships. This work was supported by the Russian Ministry of Education and Science within the base portion of the state task to Samara State Technical University (project no. 1151) and supported by the Russian Foundation for Basic Research (project no. 16-01-00249-a). Each author has participated in the article concept development and in the manuscript writing. The authors are absolutely responsible for submitting the final manuscript in print. Each author has approved the final version of manuscript. The authors have not received any fee for the article.

\section{ORCIDs}

Vladimir P. Radchenko: http://orcid.org/0000-0003-4168-9660

Aleksey Yu. Kurov: http://orcid.org/0000-0002-3791-3829

\section{REFERENCES}

1. Birger I. A. Ostatochnye napryazheniya [Residual stresses]. Moscow, Mashgiz, 1963, 262 pp. (In Russian)

2. Pavlov V. F., Kirpichev V. A., Ivanov V. B. Ostatochnye napryazheniya $i$ soprotivlenie ustalosti uprochnennykh detaley s kontsentratorami napryazheniy [Residual Stresses and Fatigue Resistance of Hardened Parts with Stress Concentrators]. Samara, Samara Scientific Center, Russian Academy of Sciences, 2008, 64 pp. (In Russian)

3. Ivanov S. I., Pavlov V. F., Minin B. V., Kirpichev V. A., Kocherov E. P., Golovkin V. V. Ostatochnye napriazheniia i soprotivlenie ustalosti vysokonagruzhennykh rez'bovykh detalei [Residual stresses and fatigue resistance of heavily threaded parts]. Samara, Samara Scientific Center, Russian Academy of Sciences, 2015, 170 pp. (In Russian)

4. Kravchenko B. A., Krutsilo V. G., Gutman G. N. Termoplasticheskoe uprochnenie - rezerv povysheniya prochnosti i nadezhnosti detaley mashin [A Thermoplastic Hardening as a Reserve of the Increase of Strength and Reliability of Machine Parts]. Samara, Samara State Technical University, 2000, 216 pp. (In Russian)

5. Sulima G. N., Shuvalov V. A., Iagodkin Yu. D. Poverkhnostnyi sloi i ekspluatatsionnye svoistva detalei mashin [Surface layer and performance of machine parts]. Moscow, Mashinostroenie, 1988, 240 pp. (In Russian)

6. Markov A. I. Ul'trazvukovaia obrabotka materialov [Ultrasonic Processing of Materials]. Moscow, Mashinostroenie, 1989, 237 pp. (In Russian)

7. Nozhnitskii Iu. A., Fishgoit A. V., Tkachenko R. I., Teplova S. V. Development and application of new GTE parts hardening methods based on the plastic deformation of the surface layers, Vestnik dvigatelestroeniia, 2006, no. 2, pp. 8-16 (In Russian).

8. Brockman R. A., Braisted W. R., Olson S. E., Tenaglia R. D., Clauer A. H., Langer K., Shepard M. J. Prediction and characterization of residual stresses from laser shock peening, International Journal of Fatigue, 2012, vol.36, no. 1, pp. 96-108. doi: 10.1016/j.ijfatigue. 2011.08 .011$.

9. Dai K., Shaw L. Analysis of fatigue resistance improvements via surface severe plastic deformation, International Journal of Fatigue, 2008, vol.30, no. 8, pp. 1398-1408. doi: 10.1016/j.ijfatigue.2007.10.010.

10. James M. N., Hughes D. J., Chen Z., Lombard H., Hattingh D. G., Asquith D., Yates J. R., Webster P. J. Prediction and characterization of residual stresses from laser shock peening, Engineering Failure Analysis, 2007, vol.14, no.2, pp. 384-395. doi:10.1016/j. engfailanal.2006.02.011.

11. Majzoobi G. H., Azadikhah K., Nemati J. The effects of deep rolling and shot peening on fretting fatigue resistance of Aluminum-7075-T6, Materials Science and Engineering A, 2009, vol.516, no.1-2, pp. 235-247. doi: 10.1016/j.msea.2009.03.020.

12. Soady K. A. Life assessment methodologies incoroporating shot peening process effects: Mechanistic consideration of residual stresses and strain hardening: Part 1 - Effect of shot peening on fatigue resistance, Materials Science and Technology (United Kingdom), 2013, vol. 29, no. 6, pp. 637-651. doi: 10.1179/1743284713Y.0000000222. 
13. Terres M. A., Laalai N., Sidhom H. Effect of nitriding and shot-peening on the fatigue behavior of $42 \mathrm{CrMo} 4$ steel: Experimental analysis and predictive approach, Materials and Design, 2012, vol. 35, pp. 741-748. doi: 10.1016/j.matdes.2011.09.055.

14. Pavlov V. F., Stoljarov A. K., Vakuljuk V. S., Kirpichev V. A. Raschet ostatochnykh napriazhenii $v$ detaliakh s kontsentratorami napriazhenii po pervonachal'nym deformatsiiam [Calculation of residual stresses in parts with stress concentrators by initial deformations]. Samara, Samara Scientific Center, Russian Academy of Sciences, 2008, 124 pp. (In Russian)

15. Vakuljuk V. S. Investigation of influence of thickness hardened layer on the redisiual stresses in basin concentrator using initial strain, Vestn. Samar. Gos. Tekhn. Univ. Ser. Fiz.-Mat. Nauki [J. Samara State Tech. Univ., Ser. Phys. \& Math. Sci.], 2010, no. 1(20), pp. 222-225 (In Russian). doi: 10.14498/vsgtu782.

16. Sazanov V. P., Chirkov A. V., Samoilov V. A., Larionova Yu. S. Modeling of redistribution of residual stresses in the cylindrical specimens after advancing surface plastic forming, Vestnik Samarskogo gosudarstvennogo aerokosmicheskogoo universiteta, 2011, no. 3(27), pp. 171-174 (In Russian).

17. Sazanov V. P., Afanas'eva O. S., Mikhal'kina S. A., Anokhin D. V. The use of finite element modeling to determining the initial deformations in the surface hardened workpiece layer, In: Proceedings of the Tenth All-Russian Scientific Conference with international participation (24-28 May 2016). Part 1, Matem. Mod. Kraev. Zadachi. Samara, Samara State Technical Univ., 2016, pp. 195-200 (In Russian).

18. Sazanov V. P., Vakuljuk V. S., Mikhalkina S. A., Surgutanov N. A., Kocherova E. E. Radial initial deformations influence on residual stresses distribution in a surface hardened cylinder, Izvestiia Samarskogo nauchnogo tsentra Rossiiskoi akademii nauk, 2014, vol.16, no. 4, pp. 163-167 (In Russian).

19. Radchenko V. P., Saushkin M. N. Polzuchest' i relaksatsiya ostatochnykh napryazheniy $v$ uprochnonnykh konstruktsiyakh [Creep and Relaxation of Residual Stresses in Hardened Structures]. Moscow, Mashinostroenie-1, 2005, 226 pp. (In Russian)

20. Saushkin M. N., Radchenko V. P., Pavlov V. F. Method of calculating the fields of residual stresses and plastic strains in cylindrical specimens with allowance for surface hardening anisotropy, J. Appl. Mech. Tech. Phys., 2011, vol.52, no. 2, pp. 303-310. doi: 10.1134/ S0021894411020180.

21. Radchenko V. P., Pavlov V. Ph., Saushkin M. N. Investigation of surface plastic hardening anisotropy influence on residual stresses distribution in hollow and solid cylindrical specimens, PNRPU Mechanics Bulletin, 2015, no.1, pp. 130-147 (In Russian). doi: 10.15593/perm.mech/2015.1.09.

22. Saushkin M. N., Kurov A. Yu. Finite element modeling of residual stress distribution in solid hardened cylindrical samples and samples with semicircular notch, Vestn. Samar. Gos. Tekhn. Univ. Ser. Fiz.-Mat. Nauki [J. Samara State Tech. Univ., Ser. Phys. \& Math. Sci.], 2011, no. 3(24), pp. 72-78 (In Russian). doi: 10.14498/vsgtu963.

23. Saushkin M. N., Kurov A. Yu. Analysis of stress state in semicircular profile notches after preliminary surface plastic deformation of solid cylindrical specimens, Vestn. Samar. Gos. Tekhn. Univ. Ser. Fiz.-Mat. Nauki [J. Samara State Tech. Univ., Ser. Phys. \& Math. Sci.], 2012, no. 1(26), pp. 133-140 (In Russian). doi : 10.14498/vsgtu1039.

Received 19/X/2016; received in revised form $28 / \mathrm{XI} / 2016$; accepted 09/XII/2016. 